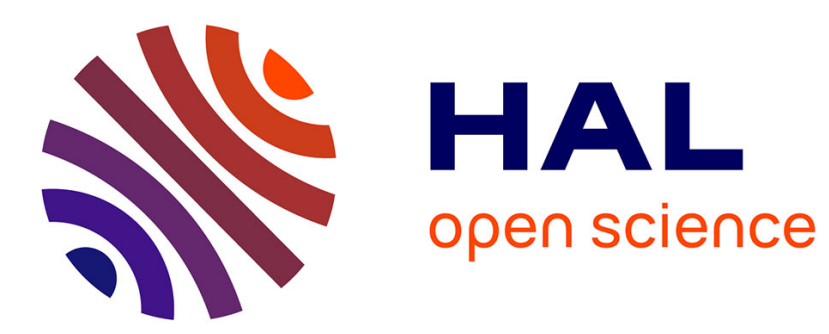

\title{
Exact control of genetic networks in a qualitative framework: the bistable switch example \\ Madalena Chaves, Jean-Luc Gouzé
}

\section{To cite this version:}

Madalena Chaves, Jean-Luc Gouzé. Exact control of genetic networks in a qualitative framework: the bistable switch example. Automatica, 2011, 47 (6), pp.1105-1112. 10.1016/j.automatica.2011.01.071 . hal-00848406

\section{HAL Id: hal-00848406 https://inria.hal.science/hal-00848406}

Submitted on 7 Aug 2013

HAL is a multi-disciplinary open access archive for the deposit and dissemination of scientific research documents, whether they are published or not. The documents may come from teaching and research institutions in France or abroad, or from public or private research centers.
L'archive ouverte pluridisciplinaire HAL, est destinée au dépôt et à la diffusion de documents scientifiques de niveau recherche, publiés ou non, émanant des établissements d'enseignement et de recherche français ou étrangers, des laboratoires publics ou privés. 
This is a preliminary version of the article published as:

M. Chaves and J.-L. Gouzé, Automatica, 47, pp. 1105-1112, 2011.

\title{
Exact control of genetic networks in a qualitative framework: the bistable switch example*
}

\author{
Madalena Chaves Jean-Luc Gouzé \\ COMORE, INRIA, 2004 Route des Lucioles, BP 93, 06902 Sophia Antipolis, France \\ madalena.chaves@inria.fr, jean-luc.gouze@inria.fr
}

\begin{abstract}
A qualitative method to control piecewise affine differential systems is proposed and explored for application to genetic regulatory networks. This study considers systems whose outputs and inputs are of a qualitative form, well suited to experimental devices: the measurements indicate whether the variables are "strongly" or "weakly" expressed, that is, only the region of the state space where trajectories evolve at each instant can be known. The control laws are piecewise constant functions in each region and in time, and are only allowed to take three qualitative values corresponding to no control $(u=1)$, high synthesis rates $\left(u=u_{\max }\right)$ or low synthesis rates $\left(u=u_{\min }\right)$. The problems of controlling the bistable switch to each of its steady states is considered. Exact solutions are given to asymptotically control the system to either of its two stable steady states. Two approximate solutions are suggested to the problem of controlling the system to the unstable steady state: either control to a neighborhood of the state, or in the form of a periodic cycle that passes through the state.
\end{abstract}

\section{Introduction}

The internal regulation of an organism, cellular growth, cellular division or differentiation, are all phenomena involving changes and readjustments in the metabolic and genetic networks. Recent progress in the understanding of cellular mechanisms is centered on experimentally tinkering with the system, to obtain a desired dynamical behaviour. The first generation of synthetic biology experiments have shown that molecular components can be assembled and tuned to reproduce desired dynamics: a bistable system [10], an oscillatory network [8], or a mammalian tunable oscillator [21] have been designed and constructed in the laboratory.

Many interesting theoretical control problems arise from the analysis of biological regulatory systems [20]. These systems have constraints regarding the type of available measurements (outputs) and the possible ways to act on the system (inputs). In particular, the solutions to control problems in biological networks will be relevant to the biologist only if the control laws can be implemented in the laboratory, using molecular components. Classical control methods [19] (often based on the linearization of the system) rely on the complete knowledge of (some of) the state variables of the system, at regular time intervals. However, detailed quantitative knowledge is often difficult or very expensive to obtain in the laboratory.

For these reasons, we will consider the class of piecewise affine (PWA) differential systems, which are based on a qualitative description of the system, and can be easily compared with the experimental data obtained from gene and protein expression. This class of systems has been widely used for the analysis of genetic regulatory networks $[12,6,3,5]$, and some control problems have already been studied in [9]. Recently, [7] use piecewise constant additive inputs to design a control that generates a sustained periodic orbit in a PWA damped oscillator. Control questions for affine systems on polytopes

*This work was supported in part by the INRIA-INSERM initiative action ColAge and ANR GeMCo (French national research agency). 
are studied in [14], which considers the problem of reaching a desired facet using piecewise-affine state feedback; multi-affine systems are studied [2], with an application to biological networks. It should be emphasized that both [14] and [2] need to use the precise value of the variables at each instant. Another study uses a probabilistic controllability analysis technique from hybrid systems to find an initial state set that can be controlled to the desired target [1].

This paper focuses on the 2-dimensional bistable system and considers the following problem: control the system to a given target, using only qualitative knowledge on the state variables and applying qualitative control. The general control problem for $n$-dimensional systems, using only constant control in each regular domain, has been studied in [9], including the problem of making a regular region invariant, or controlling the system through a given face. This general framework is, however, not sufficiently detailed to allow for fine-tuned control: the solution of some of the problems posed here (see P2 or P3 below) requires additional assumptions and could not be solved using only the general methods from [9]. Motivated by the recent work on experimental design and synthetic biology [10, 8, 21], it will be assumed that synthesis rates can be regulated by the biologist (for instance, with the introduction of a plasmid containing an inducible promoter of a given gene). It will also be assumed that the control law is piecewise constant and ranges over a finite set of values: small or large synthesis rates, or no control. For the example of the bistable switch (Section 2), exact solutions are presented to control the system to either of the stable steady states (Sections 4 and 5). The problem of controlling the system to the unstable steady state has two approximate solutions (Section 6): either controlling the system to a neighborhood of the state, or generating a periodic solution passing through the state. Finally (Section 7), we provide some remarks on how to apply these results to larger systems and a comparison to the experiments in [10].

\section{The bistable switch}

The bistable switch is a well known model of an interaction between two genes. Mathematical models of the bistable switch are characterized by the existence of two stable steady states (or two stable modes), representing two distinct outcomes of the biological system [4]. Both modes are stable to small variations in the expression of the genes. Such behaviour has been observed experimentally in several systems, such as the lac operon in the bacteria Escherichia coli, a group of genes which are repressed in the presence of glucose but transcribed in the absence of glucose and presence of lactose [15, 17]; or the phage $\lambda$ virus that infects $E$. coli and can be found in two possible states, corresponding to a dormant (lysogentic) state or a reproducible (lytic) mode [18]. Moreover, a bistable system has been experimentally designed and assembled in the laboratory, from "single pieces" [10], thus showing that such a mechanism may indeed be the working principle of many biological phenomena.

The classical model is composed of two variables which mutually inhibit each other, and are degraded at a constant rate. The inhibition functions are of sigmoidal type, with a threshold concentration defining the value of influence of one variable on the other. Let $x=\left(x_{1}, x_{2}\right)^{\prime} \in \mathbb{R}_{\geq 0}^{2}$, and consider $\kappa_{i}, \gamma_{i}, \theta_{i}>0(i=1,2)$, where $\kappa_{i}$ denote production rates, $\gamma_{i}$ denote the degradation rate constants, and $\theta_{i}$ the threshold concentrations. The system can be written:

$$
\begin{aligned}
& \dot{x}_{1}=\kappa_{1} h^{-}\left(x_{2}, \theta_{2}, n_{2}\right)-\gamma_{1} x_{1}, \\
& \dot{x}_{2}=\kappa_{2} h^{-}\left(x_{1}, \theta_{1}, n_{1}\right)-\gamma_{2} x_{2} .
\end{aligned}
$$

where $h^{-}(x, \theta, n)=\theta^{n} /\left(x^{n}+\theta^{n}\right)$. It is easy to check (for instance by drawing the nullclines) that, if the parameters satisfy $n_{1}, n_{2} \gg 1$ and:

$$
\theta_{i}<\frac{\kappa_{i}}{\gamma_{i}}
$$

system (1) has two stable steady states, corresponding to high $x_{1}$, low $x_{2}$ (P1) or low $x_{1}$, high $x_{2}(\mathrm{P} 2)$, that is, one of the variables is at a high concentration and represses the other. A third unstable steady state (P3) exists, corresponding to both variables close to their threshold concentrations.

More generally, we are interested in a qualitative description of the bistable switch, corresponding to the case $n_{i} \rightarrow \infty$ where the sigmoidal functions $h^{-}$become step functions. Without loss of generality, 
we will consider only the case when the system is defined inside the (invariant) set $\left[0, \kappa_{1} / \gamma_{1}\right] \times\left[0, \kappa_{2} / \gamma_{2}\right]$ :

$$
\begin{aligned}
& \dot{x}_{1}=\kappa_{1} s^{-}\left(x_{2}, \theta_{2}\right)-\gamma_{1} x_{1}, \\
& \dot{x}_{2}=\kappa_{2} s^{-}\left(x_{1}, \theta_{1}\right)-\gamma_{2} x_{2} .
\end{aligned}
$$

with

$$
s^{-}(r, \theta)= \begin{cases}1, & r<\theta \\ 0, & r>\theta\end{cases}
$$

This class of piecewise affine systems (PWA) was first introduced by L. Glass [12], and is widely used for modeling genetic regulatory networks $[12,6,3,5]$. Step functions are not defined at threshold points, but solutions of the system "across" or "along" a threshold can still be defined in the sense of Filippov, as the solutions of differential inclusions. See for instance [3] for an analysis of solutions of PWA systems. Throughout this paper, the definitions given in this reference will be adopted. For self containment of the paper, a brief summary of some special cases is provided in the appendix. In this case, there are also two stable steady states, $\mathrm{P} 1$ and $\mathrm{P} 2$, and an unstable Filippov equilibrium point, P3, analogous to a saddle point:

$$
P 1=\left(\frac{\kappa_{1}}{\gamma_{1}}, 0\right), \quad P 2=\left(0, \frac{\kappa_{2}}{\gamma_{2}}\right), \quad P 3=\left(\theta_{1}, \theta_{2}\right) .
$$

It is easy to see that the region: $\Omega=\left[0, \kappa_{1} / \gamma_{1}\right] \times\left[0, \kappa_{2} / \gamma_{2}\right]$ is forward invariant for system (3), so from now on, we consider only solutions evolving on $\Omega$. The dynamics of system (4) can be divided into four regions, or regular domains, where the vector field is uniquely defined:

$$
\begin{aligned}
& B_{00}=\left\{x \in \mathbb{R}_{\geq 0}^{2}: 0<x_{1}<\theta_{1}, 0<x_{2}<\theta_{2}\right\} \\
& B_{01}=\left\{x \in \mathbb{R}_{\geq 0}^{2}: 0<x_{1}<\theta_{1}, \theta_{2}<x_{2}<\kappa_{2} / \gamma_{2}\right\} \\
& B_{10}=\left\{x \in \mathbb{R}_{\geq 0}^{2}: \theta_{1}<x_{1}<\kappa_{1} / \gamma_{1}, 0<x_{2}<\theta_{2}\right\} \\
& B_{11}=\left\{x \in \mathbb{R}_{\geq 0}^{2}: \theta_{1}<x_{1}<\kappa_{1} / \gamma_{1}, \theta_{2}<x_{2}<\kappa_{2} / \gamma_{2}\right\} .
\end{aligned}
$$

In addition, there are also switching domains, where the system is defined only as a differential inclusion, corresponding to the segments where each of the variables is at a threshold $\left(x_{i}=\theta_{i}\right.$ and $\left.x_{j} \in\left[0, \kappa_{j} / \gamma_{j}\right]\right)$.

\section{Measurements and control}

The solution to the problem of controlling a system is typically based on the notions of input and output. (see [19], for reference). The output consists of measurements of some, or a combination, of the variables that indicate the current state of the system, say $h(x(t))=x_{2}(t)$. Classical control methods need to know this function at each instant $t$ (or at regular time intervals, with a minimum frequency). For genetic regulatory networks, the measurements are often of a qualitative form, indicating only if a gene is strongly or weakly expressed (see [16] for a review of experimental methods, such as microarrays or Western blots; see also [11]). In the qualitative framework introduced in Section 2, this means that only the relative position of the variables with respect to the threshold are known, that is, the available measurements are of the form $s^{+}\left(x_{2}(t), \theta_{2}\right) \in\{0,1\}$, where $s^{+}(x, \theta)=1-s^{-}(x, \theta)$.

The input represents the actions that a user (here a biologist) is able to exert on the system. From an experimental point of view, one common manipulation is to change the synthesis rate of messenger RNA by addition of a plasmid. Plasmids are basically short rings of DNA that can be specially constructed to include an inducible promoter of the gene under study [10,8], and thus the transcription rate may be externally regulated by adding different concentrations of the inducer. Therefore, it is reasonable to suppose that the inputs will act on the synthesis rates. To reflect the qualitative data and experimental constraints, it will be assumed that inputs are bounded, piecewise constant functions that can depend both on time and space. The dependence of $u$ on the space is further constrained to the type of 
measurements: $X_{i}=s^{+}\left(x_{i}(t), \theta_{i}\right) \in\{0,1\}, i=1,2$. In addition, it will be assumed that only three qualitative control values can reasonably be implemented:

$$
u(t, X): \mathbb{R}_{\geq 0} \times\{0,1\}^{2} \rightarrow\left\{u_{\min }, 1, u_{\max }\right\},
$$

where $u=1$ corresponds to no control, $u=u_{\min }$ corresponds to low synthesis control, and $u=u_{\max }$ corresponds to high synthesis control. The values $u_{\min }<1<u_{\max }$ should satisfy the conditions (8), (9) given below. Since (3) is a 2-dimensional system, inputs will be single-valued. The system with inputs can thus be written:

$$
\begin{aligned}
& \dot{x}_{1}=u \kappa_{1} s^{-}\left(x_{2}, \theta_{2}\right)-\gamma_{1} x_{1} \\
& \dot{x}_{2}=u \kappa_{2} s^{-}\left(x_{1}, \theta_{1}\right)-\gamma_{2} x_{2} .
\end{aligned}
$$

In Sections 4-6, the following question will be studied: find a control law $u=u(t, X)$ (with the properties stated above) such that the system (4) is globally convergent to the point $\mathrm{P}$, where $P \in\{P 1, P 2, P 3\}$. To do this, a standard approach is to linearize the system and construct a local solution, but this does not provide a satisfactory answer in the case of genetic networks with qualitative measurements. In spite of these difficulties and constraints, we have been able to construct complete global solutions to exactly control the system (4) to $P \in\{P 1, P 2\}$. Exact solutions could not be found for the problem of controlling to the unstable state $P=P 3$; instead two alternative solutions are suggested to bring the system close to that point.

For system (4), a limited number of control strategies are available within each of the four regions:

$B_{11}$ : no control is possible, the trajectories will converge towards the origin;

$B_{01}$ : the trajectories converge towards $\left(0, u \frac{\kappa_{2}}{\gamma_{2}}\right)$, so it is possible to change the focal point, along the $x_{2}$-axis;

$B_{10}$ : the trajectories converge towards $\left(u \frac{\kappa_{1}}{\gamma_{1}}, 0\right)$, so it is possible to change the focal point, along the $x_{1}$-axis;

$B_{00}$ : the trajectories converge towards $\left(u \frac{\kappa_{1}}{\gamma_{1}}, u \frac{\kappa_{2}}{\gamma_{2}}\right)$, so it is possible to change the focal point along the line $x_{2}=\phi\left(x_{1}\right)=\frac{\kappa_{2}}{\gamma_{2}} \frac{\gamma_{1}}{\kappa_{1}} x_{1}$.

In each region of the phase plane a trajectory starting at $\left(x_{10}, x_{20}\right)$ evolves along a curve of the form:

$$
x_{2}=\sigma\left(x_{1} ; u\right)=M_{2}+\left(x_{20}-M_{2}\right)\left(\frac{x_{1}-M_{1}}{x_{10}-M_{1}}\right)^{\frac{\gamma_{2}}{\gamma_{1}}}
$$

where $M_{i} \in\left\{0, u \kappa_{i} / \gamma_{i}\right\}$. We also have:

$$
x_{1}=\sigma^{-1}\left(x_{2} ; u\right)=M_{1}+\left(x_{10}-M_{1}\right)\left(\frac{x_{2}-M_{2}}{x_{20}-M_{2}}\right)^{\frac{\gamma_{1}}{\gamma_{2}}} .
$$

For each $u \geq 1$, there is a curve (a separatrix) dividing $B_{00}$ into two regions (above and below the separatrix), from which trajectories will reach $B_{01}$ and $B_{10}$, respectively. For the case $u=1$, these two regions belong to the basins of attraction for $\mathrm{P} 2$ and $\mathrm{P} 1$, respectively. The separatrix has the form:

$$
\alpha\left(x_{1} ; u\right)=u \frac{\kappa_{2}}{\gamma_{2}}-\left(u \frac{\kappa_{2}}{\gamma_{2}}-\theta_{2}\right)\left(\frac{u \frac{\kappa_{1}}{\gamma_{1}}-x_{1}}{u \frac{\kappa_{1}}{\gamma_{1}}-\theta_{1}}\right)^{\frac{\gamma_{2}}{\gamma_{1}}}
$$

The points $\left(x_{1}, \alpha\left(x_{1} ; u\right)\right)$, with $u \geq 1$, represent the locus of any trajectory in $B_{00}$ that reaches the point $\left(\theta_{1}, \theta_{2}\right)$.

\section{Assumptions}

In addition to conditions (2), the following hypotheses on the parameters will be considered:
H1 $\quad \frac{\theta_{2}}{\theta_{1}}>\frac{\kappa_{2}}{\kappa_{1}} \frac{\gamma_{1}}{\gamma_{2}} \quad \Leftrightarrow \quad \phi\left(\theta_{1}\right)<\theta_{2} ;$
H2 $\frac{\theta_{2}}{\theta_{1}}<\frac{\kappa_{2}}{\kappa_{1}}$. 
Hypothesis $\mathrm{H} 1$ implies that the line $x_{2}=\phi\left(x_{1}\right)$ of possible focal points for $B_{00}$ is below the unstable point $\left(\theta_{1}, \theta_{2}\right)$. Hypothesis $\mathrm{H} 2$ guarantees that a value $x_{1}^{*}>0$ exists such that $\alpha\left(x_{1}^{*} ; u_{\max }\right)=0$, for $u_{\max }$ sufficiently large (see Lemma 1 below). Note that H1 and H2 imply that $\frac{\gamma_{1}}{\gamma_{2}}<1$. The upper and lower bounds $u_{\min }, u_{\max }$ are defined as follows $\left(\delta<\frac{\theta_{1} \kappa_{2}}{\theta_{2} \kappa_{1}}-1\right.$ and $\varepsilon>0$ is small):

$$
u_{\max }>\max \left\{1, \frac{(1+\delta) \gamma_{2} \frac{\theta_{2}}{\kappa_{2}}-\gamma_{1} \frac{\theta_{1}}{\kappa_{1}}}{\delta}\right\}
$$

and

$$
0<u_{\min }<\min \left\{\frac{\gamma_{1}}{\kappa_{1}} \theta_{1}, \frac{\gamma_{2}}{\kappa_{2}} \theta_{2},(1-\varepsilon) x_{1}^{*} \frac{\gamma_{1}}{\kappa_{1}}\right\}
$$

Finally, we remark that hypothesis H1 is only a geometric choice; the complementary case, $\frac{\theta_{2}}{\theta_{1}}<\frac{\kappa_{2}}{\kappa_{1}} \frac{\gamma_{1}}{\gamma_{2}}$, can be treated in an analogous form, with $\frac{\theta_{2}}{\theta_{1}}>\frac{\kappa_{2}}{\kappa_{1}}$, and requiring that $\alpha$ crosses the $y$-axis, i.e., there exists $x_{2}^{*}$ such that $x_{2}^{*}=\alpha\left(0 ; u_{\max }\right)$. The next Lemma summarizes some useful facts on the separatrix function and also justifies the choice of $\mathrm{H} 2$.

Lemma 1 Assume that H1 and H2 hold and consider the function $\alpha: \mathbb{R}_{\geq 0} \times\left(\max _{i=1,2}\left\{\gamma_{i} \frac{\theta_{i}}{\kappa_{i}}\right\}, \infty\right) \rightarrow$ $\mathbb{R}_{\geq 0}$, given by (7). Then:

a. For each fixed $u, \alpha$ is increasing w.r.t. the variable $x$;

b. Define the function $x_{10}^{*}=x_{1}^{*}(u)$ by $\alpha\left(x_{10}^{*} ; u\right)=0$. Assume that H2 holds. Then, for sufficiently large $u, x_{10}^{*}$ is increasing and satisfies $\lim _{u \rightarrow \infty}=\theta_{1}-\theta_{2} \frac{\kappa_{1}}{\kappa_{2}}$.

Proof. Part (a) is immediate from the fact that $u$ is larger than $\max _{i=1,2}\left\{\gamma_{i} \theta_{i} / \kappa_{i}\right\}$ and analysis of the expression (7). With the notation $\tilde{\gamma}_{i}=\gamma_{i} \frac{\theta_{i}}{\kappa_{i}}, i=1,2$, the function $x_{1}^{*}$ can be written as follows:

$$
\begin{aligned}
x_{10}^{*}(u) & =u \frac{\kappa_{1}}{\gamma_{1}}\left[1-\left(1-\frac{\tilde{\gamma}_{1}}{u}\right)\left(\frac{1}{1-\frac{\tilde{\gamma}_{2}}{u}}\right)^{\frac{\gamma_{1}}{\gamma_{2}}}\right] \\
& :=u \frac{\kappa_{1}}{\gamma_{1}}[1-g(u)] .
\end{aligned}
$$

The limit as $u$ tends to infinity can be computed by l'Hôpital's rule, writing $x_{10}^{*}(u)=\frac{\kappa_{1}}{\gamma_{1}} \frac{1-g(u)}{\frac{1}{u}}$. To show that $x_{10}^{*}$ is increasing for sufficiently large $u$, we will show that there exists $u^{*}$ such that $g(u)$ is increasing for $u>u^{*}$ and $\lim _{u \rightarrow \infty} g(u)=1$. The derivative of $g$ has the form:

$$
\frac{d g}{d u}=\frac{\tilde{\gamma}_{1}}{u^{2}}\left(\frac{u}{u-\tilde{\gamma}_{2}}\right)^{\frac{\gamma_{1}}{\gamma_{2}}}\left[1-\frac{\tilde{\gamma}_{2}}{\tilde{\gamma}_{1}} \frac{\gamma_{1}}{\gamma_{2}} \frac{u-\tilde{\gamma}_{1}}{u-\tilde{\gamma}_{2}}\right]
$$

Observe that $\mathrm{H} 1$ is equivalent to $\tilde{\gamma}_{1}<\tilde{\gamma}_{2}$, so the term $\left(u-\tilde{\gamma}_{1}\right) /\left(u-\tilde{\gamma}_{2}\right)>1$ is decreasing. Now, using H2, define

$$
0<\delta<\frac{\theta_{1}}{\theta_{2}} \frac{\kappa_{2}}{\kappa_{1}}-1, \text { and } u^{*}=\frac{(1+\delta) \tilde{\gamma}_{2}-\tilde{\gamma}_{1}}{\delta} .
$$

It follows that

$$
\begin{aligned}
u \geq u^{*} \Rightarrow \quad & 1-\frac{\tilde{\gamma}_{2}}{\tilde{\gamma}_{1}} \frac{\gamma_{1}}{\gamma_{2}} \frac{u-\tilde{\gamma}_{1}}{u-\tilde{\gamma}_{2}}>1-\frac{\tilde{\gamma}_{2}}{\tilde{\gamma}_{1}} \frac{\gamma_{1}}{\gamma_{2}} \frac{u^{*}-\tilde{\gamma}_{1}}{u^{*}-\tilde{\gamma}_{2}} \\
= & 1-\frac{\tilde{\gamma}_{2}}{\tilde{\gamma}_{1}} \frac{\gamma_{1}}{\gamma_{2}}(1+\delta)>1-\frac{\tilde{\gamma}_{2}}{\tilde{\gamma}_{1}} \frac{\gamma_{1}}{\gamma_{2}} \frac{\theta_{1}}{\theta_{2}} \frac{\kappa_{2}}{\kappa_{1}}=1,
\end{aligned}
$$

so $g(u)$ is indeed increasing for all $u \geq u^{*}$, and remains below 1 . This implies that the derivative of $x_{10}^{*}$ with respect to $u$ is positive, and hence the function is increasing for $u \geq u^{*}$, as we wanted to show. 


\section{Controlling to P1}

There is a complete solution to this problem, using the following piecewise constant function $u$ (recall that $\left.X_{i}=s^{+}\left(x_{i}, \theta_{i}\right)\right)$ :

$$
u(t, X(x))= \begin{cases}1, & \forall t, x \in B_{11} \cup B_{10} \cup B_{00} \\ u_{\text {min }}, & \forall t, x \in B_{01}\end{cases}
$$

Possible trajectories are shown in Fig. 1. For this problem, only assumption H1 is needed. The solution is based on the existence of a sliding mode solution on the boundary between $B_{01}$ and $B_{00}$ :

Lemma 2 Assume that hypothesis $H 1$ is satisfied. Consider system (4) with control function (10). Then, there is a sliding mode solution on the line $x_{2}=\theta_{2}$ with $x_{1}<\theta_{1}$ that will reach point P3, after a finite time $T_{P 3}$. The same result holds if $u(t, X(x))=u_{\max }$ for $x \in B_{00}$.

Proof. To analyze the system on this switching domain, note that the vector fields have opposite directions along this line, so a solution should be interpreted in the sense of Filippov:

$$
\left(\begin{array}{l}
\dot{x}_{1} \\
\dot{x}_{2}
\end{array}\right) \in \overline{\mathrm{co}}\left\{\left(\begin{array}{l}
u_{\max } \kappa_{1}-\gamma_{1} x_{1} \\
u_{\max } \kappa_{2}-\gamma_{2} x_{2}
\end{array}\right),\left(\begin{array}{c}
0-\gamma_{1} x_{1} \\
u_{\min } \kappa_{2}-\gamma_{2} x_{2}
\end{array}\right)\right\} .
$$

where $u_{\min } \kappa_{2}-\gamma_{2} x_{2}<0<u_{\max } \kappa_{2}-\gamma_{2} x_{2}$ and $-\gamma_{1} x_{1}<0<u_{\max } \kappa_{1}-\gamma_{1} x_{1}$, using (2) for $x_{1}<\theta_{1}$ and $x_{2}$ in a small neighborhood of $\theta_{2}$. Letting $y=x_{1}$ and $x=x_{2}$ in Lemma 7 , we can conclude that there is a sliding mode towards a point $\left(\bar{x}, \theta_{2}\right)$, where

$$
\bar{x}=\frac{\kappa_{1}}{\gamma_{1}}\left(\frac{\gamma_{2}}{\kappa_{2}} \theta_{2}-u_{\min }\right) \frac{u_{\max }}{u_{\max }-u_{\min }} .
$$

By H1, and for $u_{\text {min }}$ sufficiently small, $\bar{x}>\theta_{1}$, so the sliding mode reaches $\left(x_{1}, x_{2}\right)=\left(\theta_{1}, \theta_{2}\right)=\mathrm{P} 3$, after a time $t \leq T_{P 3}$, which can be estimated by letting $x_{10}=0$ and calculating $x_{1}\left(T_{P 3}\right)=\theta_{1}=\bar{x}\left(1-e^{-\gamma_{1} T_{P 3}}\right)$. Note that the result holds also if $u_{\max }=1$.

Theorem 1 Assume that hypothesis H1 is satisfied. Then system (4) with control function (10) converges to the point $P 1=\left(\kappa_{1} / \gamma_{1}, 0\right)$.

Proof. It is easy to see that any trajectory starting in $B_{10}$ will directly converge to the point P1. Any trajectory starting in $B_{11}$ will enter either $B_{10}$ or $B_{01}$ in finite time. Any trajectory starting in $B_{01}$ will reach the switching domain $x_{2}=\theta_{2}\left(0 \leq x_{1}<\theta_{1}\right)$ in finite time. Any trajectory starting in $B_{00}$ will reach either $B_{10}$ or the switching domain $x_{2}=\theta_{2}$ in finite time. Trajectories evolving from $B_{11}$ to $B_{10}$ or from $B_{00}$ to $B_{10}$ can be normally continued, according to Lemma 6 . On the switching domain $x_{2}=\theta_{2}$, solutions evolve towards the point P3, by Lemma 2. Finally, from this point trajectories can only enter $B_{10}$, since the vector fields prevent any other trajectory.

\section{Controlling to P2}

This problem can also be completely solved, based on the dynamics in the region $B_{00}$. Recall that, in this region, the focal point may be moved only along the line $x_{2}=\phi\left(x_{1}\right)$. In particular, using the two qualitative control values, the new focal point can be either near the origin $\left(\phi_{00, \min }\right)$ or towards large values $\left(\phi_{00, \max }\right)$ :

$$
\phi_{00, m}=\left(u_{m} \frac{\kappa_{1}}{\gamma_{1}}, u_{m} \frac{\kappa_{2}}{\gamma_{2}}\right),
$$

with $m \in\{\min , \max \}$. In region $B_{10}$, the control should be set to $u=u_{\min }$ to prevent convergence to $\mathrm{P} 1$, while in $B_{01}$, it is clear that we want $u=1$, since it contains P2. Different dynamics can be 


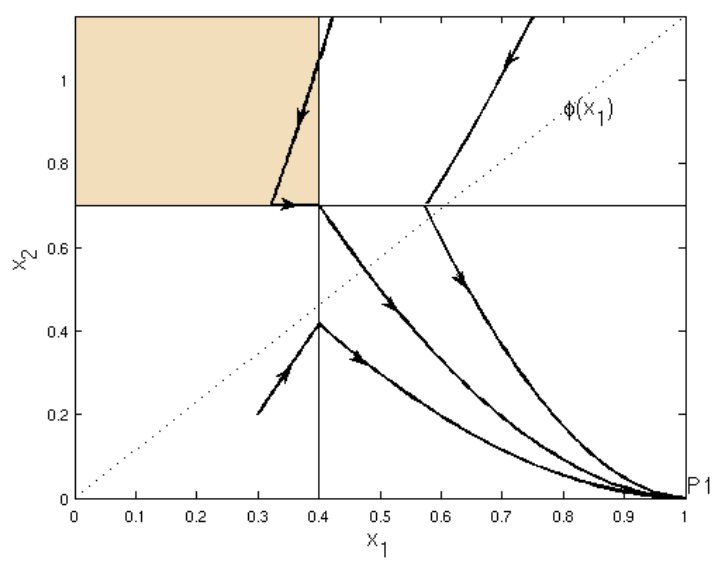

Figure 1: Controlling to P1 from each of the four regions. Control is $u=u_{\min }$ in dark shaded region and $u=1$ otherwise.

generated in $B_{00}$, by choosing $u=u_{\max }$ or $u=u_{\min }$ : in each case, trajectories are shown to converge towards different reference points (see Section 5.1, below). Studying the dynamics in $B_{00}$ with $u_{\text {min }}$ (Lemma 5) shows that any trajectory can be driven to the neighborhood of $\phi_{00, \min }$ in finite time. In particular, note that points in this neighborhood of $\phi_{00, \text { min }}$ are above the separatrix $\alpha$ and can thus be driven to the boundary $B_{00} / B_{01}$ under $u=u_{\max }$ (see Lemma 1). A possible trajectory is sketched in Fig. 2.

This suggests a piecewise constant control based on a subdivision of the region $B_{00}$. However, such a subdivision may be difficult to detect experimentally, because only the relative positions of the variables with respect to the thresholds are known. A control design more suitable for biological application will use a function that is constant within each original qualitative region, but is allowed to switch values after finite time intervals, as follows:

$$
u(t, X(x))= \begin{cases}1, & \forall t, x \in B_{11} \cup B_{01} \\ u_{\min }, & \forall t, x \in B_{10} \\ u_{\min }, & t \in\left[0, T_{1}\right), x \in B_{00} \\ u_{\max }, & t \in\left[T_{1}, \infty\right), x \in B_{00}\end{cases}
$$

where $T_{1}$ is sufficiently large. The main result is:

Theorem 2 Assume H1 and H2 hold. Then, the point P2 is globally asymptotically stable for the system (4) defined in the invariant set $\Omega=\left[0, \kappa_{1} / \gamma_{1}\right] \times\left[0, \kappa_{2} / \gamma_{2}\right]$, with the control law (11).

Proof. First, it is clear that trajectories with initial condition in $B_{01}$ immediately converge to point P2. Next, trajectories with initial condition in $B_{11}$ will enter either $B_{01}$ or $B_{10}$, in finite time (by natural continuation of solutions, as described in Lemma 6). The minimum time to guarantee that all trajectories with initial condition in $B_{11}$ reach its boundary is:

$$
T_{11}=\max _{i=1,2}\left\{\frac{1}{\gamma_{i}} \log \frac{\kappa_{i}}{\theta_{i} \gamma_{i}}\right\} .
$$

Trajectories that start in $B_{10}$ will reach the boundary between $B_{00}$ and $B_{10}$. The minimum time to guarantee that all trajectories with initial condition in $B_{01}$ reach $B_{00}$ is:

$$
T_{01}=\frac{1}{\gamma_{1}} \log \frac{\frac{\kappa_{1}}{\gamma_{1}}-u_{\min } \frac{\kappa_{1}}{\gamma_{1}}}{\theta_{1}-u_{\min } \frac{\kappa_{1}}{\gamma_{1}}} .
$$

Therefore, after a time $T_{11}+T_{01}$ we are certain that all trajectories starting inside the set $\Omega$ have entered either $B_{01}$ or $B_{00}$. By Lemma 5 , trajectories starting in $B_{00}$ with $u=u_{\min }$ reach a neighborhood of 


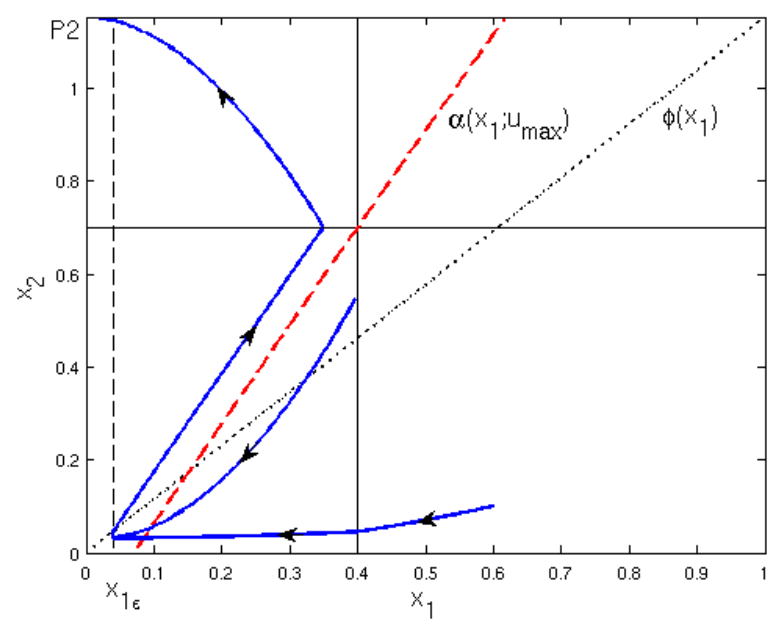

Figure 2: Controlling to P2, according to Theorem 2. In the time interval $\left[0, T_{1}\right), u=u_{\min }$ and trajectories evolve towards $\phi_{00, \min }$, above the separatrix $\alpha\left(x_{1} ; u_{\max }\right)$. Next, the control is switched to $u_{\max }$ and trajectories evolve towards the box $B_{01}$ and to point P2.

the point $\phi_{00, \text { min }}$ after time $T_{\varepsilon}$. Therefore, for any time $t>T_{1}:=T_{11}+T_{01}+T_{\varepsilon}$, any trajectory with initial condition in $\Omega$ and control law (11) is in a neighborhood of $\phi_{00, \min }$, with $x_{1}(t)<x_{1 \varepsilon}$. At time $T_{1}$, the control on $B_{00}$ is switched to $u_{\max }$. Then, by Lemma 3 , the trajectories will enter the box $B_{01}$ in finite time.

\subsection{Dynamics on $B_{00}$}

In the case $u>1$ in $B_{00}$, the separatrix $\alpha$ precisely divides this box into two regions that evolve towards either of the neighbour boxes.

Lemma 3 Assume $H 1$ and $H 2$ hold. Let $u=u_{\max }$ in $B_{00}$. The trajectories with initial state $\left(x_{10}, x_{20}\right) \in$ $B_{00}$ with $x_{20}>\alpha\left(x_{10} ; u_{\max }\right)$, reach the boundary between $B_{00}$ and $B_{01}$ in finite time $T_{\alpha}$, at a point $\left(\sigma^{-1}\left(\theta_{2} ; u_{\max }\right), \theta_{2}\right)$, with $\sigma^{-1}\left(\theta_{2} ; u_{\max }\right)<\theta_{1}$.

Proof. Let $\left(x_{1}(t), x_{2}(t)\right), x_{2}(t)=\sigma\left(x_{1}(t) ; u_{\max }\right)$ represent the trajectory with initial condition $\left(x_{10}, x_{20}\right)$ with $x_{20}>\alpha\left(x_{10} ; u_{\max }\right)$, on $B_{00}$ with control $u_{\max }$. Recall that $x_{2}(t)=\alpha\left(x_{1}(t) ; u_{\max }\right)$ represents the solution with initial condition $\left(x_{10}, \alpha\left(x_{10} ; u_{\max }\right)\right)$ passing through the point $\left(\theta_{1}, \theta_{2}\right)$. Then, by uniqueness of solutions, $\sigma\left(x_{1}(t) ; u_{\max }\right)>\alpha\left(x_{1}(t) ; u_{\max }\right)$. Therefore, defining $x_{1}^{*}$ by $\theta_{2}=\sigma\left(x_{1}^{*} ; u_{\max }\right)>\alpha\left(x_{1}^{*} ; u_{\max }\right)$ and since $\alpha\left(\theta_{1} ; u_{\max }\right)=\theta_{2}$ and $\alpha$ is an increasing function of $x_{1}$, we have that $x_{1}^{*}<\theta_{1}$. Since the trajectory $\left(x_{1}(t), \sigma\left(x_{1}(t) ; u_{\max }\right)\right)$ has its focal point outside $B_{00}$, it reaches the boundary between $B_{00}$ and $B_{01}$ in finite time. To estimate $T_{\alpha}$, let $x_{20}=0$ and calculate $x_{2}\left(T_{\alpha}\right)=\theta_{2}=u_{\max } \frac{\kappa_{2}}{\gamma_{2}}\left(1-e^{-\gamma_{2} T_{\alpha}}\right)$.

Lemma 4 Assume H1 and H2 hold. Let $u=u_{\max }$ in $B_{00}$ and $u=u_{\min }$ in $B_{10}$. The trajectories with initial state $\left(x_{10}, x_{20}\right) \in B_{00}$ with $x_{20}<\alpha\left(x_{10} ; u_{\max }\right)$, reach the boundary between $B_{00}$ and $B_{10}$ in finite time, and converge to a point $\left(\theta_{1}, \theta_{2}^{*}\right)$ with $\theta_{2}^{*}<\phi\left(\theta_{1}\right)$.

Proof. By uniqueness of solutions, the trajectory $\left(x_{1}(t), \sigma\left(x_{1}(t) ; u_{\max }\right)\right)$ corresponding to an initial condition $\left(x_{10}, x_{20}\right)$ with $x_{20}<\alpha\left(x_{1} ; u_{\max }\right)<\alpha\left(x_{10} ; u_{\max }\right)$, satisfies $\sigma\left(x_{1} ; u_{\max }\right)<\alpha\left(x_{1} ; u_{\max }\right)$. Therefore, for $x_{1}=\theta_{1}, \sigma\left(\theta_{1} ; u_{\max }\right)<\alpha\left(\theta_{1} ; u_{\max }\right)=\theta_{2}$. This shows that any such trajectory will reach the boundary between $B_{00}$ and $B_{10}$. On this boundary line, the vector fields from $B_{00}$ and $B_{10}$ point in opposite directions, and solutions can be defined in the sense of Filippov, for the differential inclusion:

$$
\left(\begin{array}{c}
\dot{x}_{1} \\
\dot{x}_{2}
\end{array}\right) \in \overline{\mathrm{co}}\left\{\left(\begin{array}{l}
u_{\max } \kappa_{1}-\gamma_{1} x_{1} \\
u_{\max } \kappa_{2}-\gamma_{2} x_{2}
\end{array}\right),\left(\begin{array}{c}
u_{\min } \kappa_{1}-\gamma_{1} x_{1} \\
-\gamma_{2} x_{2}
\end{array}\right)\right\} .
$$


Observe that $u_{\min } \kappa_{1}-\gamma_{1} x_{1}<0<u_{\max } \kappa_{1}-\gamma_{1} x_{1}$ in a small neighborhood of $\theta_{1}$. Also, $u_{\max } \kappa_{2}-\gamma_{2} x_{2}$ and $-\gamma_{2} x_{2}$ have opposite signs for $x_{2}<\theta_{2}$. Therefore, by Lemma 7 (with $x=x_{1}$ and $y=x_{2}$ ) and some algebra, the trajectories converge to: $x_{1}=\theta_{1}$ and

$$
x_{2}=\theta_{2}^{*}=\phi\left(\theta_{1}\right)\left(1-u_{\min } \frac{\kappa_{1}}{\gamma_{1}} \frac{1}{\theta_{1}}\right) \frac{u_{\max }}{u_{\max }-u_{\min }} .
$$

Now, it is not difficult to check that $\theta_{2}^{*}<\phi\left(\theta_{1}\right)$ whenever $u_{\max }>\frac{\gamma_{1}}{\kappa_{1}} \theta_{1}$, which always holds by (2).

Using the function $x_{10}^{*}$ defined in Lemma 1 , introduce a small quantity $\varepsilon>0$ and define a value near the focal point coordinate $\left(\phi_{00, \min }\right)_{i}$ :

$$
x_{1 \varepsilon}=(1+\varepsilon) u_{\min } \frac{\kappa_{1}}{\gamma_{1}}<x_{10}^{*}\left(u_{\max }\right) .
$$

Lemma 5 Assume $H 1$ and H2 hold. Let $u=u_{\min }$ in $B_{00}$. The trajectories with initial condition $\left(x_{10}, x_{20}\right) \in B_{00}$ converge to the point $\phi_{00, \min }$. In particular, there exists a time $T_{\varepsilon}$ such that the trajectories satisfy $x_{1}\left(t ; x_{10}\right)<x_{1 \varepsilon}$ for $t>T_{\varepsilon}$.

Proof. In this case, the focal point $\phi_{00, \min }$ is inside the box $B_{00}$. Hence, with $u=u_{\min }$, the trajectories converge asymptotically to $\phi_{00, \text { min }}$ without leaving $B_{00}$.

Since each variable evolves independently, according to a linear equation, it follows by continuity that trajectories will reach a point with $x_{1}=x_{1 \varepsilon}$ in finite time. To find an estimate for $T_{\varepsilon}$, consider a general trajectory in $B_{00}$ :

$$
x_{i}(t)=u_{\min } \frac{\kappa_{i}}{\gamma_{i}}+\left(x_{i 0}-u_{\min } \frac{\kappa_{i}}{\gamma_{i}}\right) \exp ^{-\gamma_{i} t}, \quad i=1,2
$$

with $t_{i \varepsilon}$ the time it takes the solution to go from $x_{i 0}$ to $(1+\varepsilon) u_{\min } \frac{\kappa_{i}}{\gamma_{i}}$ :

$$
t_{i \varepsilon}=\frac{1}{\gamma_{i}} \log \frac{x_{i 0}-u_{\min } \frac{\kappa_{i}}{\gamma_{i}}}{\varepsilon u_{\min } \frac{\kappa_{i}}{\gamma_{i}}} \leq \frac{1}{\gamma_{i}} \log \frac{\frac{\kappa_{i}}{\gamma_{i}}-u_{\min } \frac{\kappa_{i}}{\gamma_{i}}}{\varepsilon u_{\min } \frac{\kappa_{i}}{\gamma_{i}}}=t_{i} .
$$

Then let $T_{\varepsilon}=2\left(t_{1}+t_{2}\right)$.

\section{Controlling to P3}

To try to globally control the system to the unstable equilibrium point $\mathrm{P} 3=\left(\theta_{1}, \theta_{2}\right)$, the control function in both orthants $B_{01}$ and $B_{10}$ has to be small, to avoid convergence to P1 or P2. Similarly, the control in $B_{00}$ cannot be fixed at $u_{\min }$ for all times. Recall from Lemma 2 that $u=u_{\min }$ on $B_{01}$ and $u=u_{\max }$ on $B_{00}$ implies a sliding mode along the line $x_{2}=\theta_{2}$ until P3 is reached. However, at P3 the vectors fields prevent a switch to any of the regular domains, without guaranteeing stability: by Lemma 4, there is a sliding mode along $x_{1}=\theta_{1}$ towards a point with $x_{2}<\theta_{2}$. Based on the problems P1 and P2, two different approximated solutions to P3 are next proposed.

\subsection{Controlling to a neighborhood of P3}

Consider the control function (with $X_{i}=s^{+}\left(x_{i}, \theta_{i}\right)$ ):

$$
u(t, X(x))= \begin{cases}1, & \forall t, x \in B_{11} \\ u_{\min }, & \forall t, x \in B_{10} \cup B_{01} \\ u_{\max }, & \forall t, x \in B_{00} .\end{cases}
$$

Theorem 3 Assume H1 and H2 hold. Consider system (4) with the control law (12). Trajectories converge to a point $P 3^{*}=\left(\theta_{1}, \theta_{2}^{*}\right)$, with $\theta_{2}^{*}<\phi\left(\theta_{1}\right)$. 
Proof. Consider control law (12). Trajectories starting in $B_{11}$ hit the boundary and cross to $B_{01}$ or $B_{10}$. From Lemma 2, trajectories starting in $B_{01}$ reach the boundary and go through a sliding mode along $x_{2}=\theta_{2}, x_{1}<\theta_{1}$ which reaches P3. Trajectories starting in $B_{10}$ reach the boundary $x_{1}=\theta_{1}$. Trajectories starting in $B_{00}$ either reach $x_{2}=\theta_{2}$ or $x_{1}=\theta_{1}$.

Note that neither $x_{1}$ nor $x_{2}$ can increase from P3. This is clear from the signs of the vector fields, and using Lemma 6. From P3 the trajectories are also prevented to enter $B_{00}$ or $B_{10}$. But, from Lemma 4, in the switching domain $x_{1}=\theta_{1}, x_{2}<\theta_{2}$, there is a sliding mode where the solution evolves along $x_{1}=\theta_{1}$ towards a point with $\theta_{2}^{*}:=\bar{y}<\phi\left(\theta_{1}\right)<\theta_{2}$. Therefore, trajectories leave the point P3 along the line $x_{1}=\theta_{1}$, and converge to the point $x=\left(\theta_{1}, \theta_{2}^{*}\right)$.

It follows from this Theorem that, if the point $\left(\theta_{1}, \theta_{2}^{*}\right)$ is sufficiently close to $\mathrm{P} 3$, then it is possible to control the system to a neighborhood of $\mathrm{P} 3$, that is if $\left|\theta_{2}-\theta_{2}^{*}\right| \leq \delta$, then the system is controllable to $\mathcal{B}(P 3 ; \delta)=\left\{x \in \mathbb{R}_{\geq 0}^{2}:|x-P 3|<\delta\right\}$.

\subsection{A periodic orbit passing through P3}

More generally, it may happen that the point P3* is not really "close" to P3. The results obtained so far suggest that, by appropriately switching the input as a function of time, some control strategies can be used to force the trajectories to pass repeatedly through the same reference points, and thus generate a periodic solution. Theorem 3 shows that, under control (12), the system has a trajectory passing through the following points: $\left(x_{1 \varepsilon},(1+\varepsilon) \phi\left(x_{1 \varepsilon}\right)\right) \rightarrow\left(\sigma^{-1}\left(\theta_{2} ; u_{\max }\right), \theta_{2}\right) \rightarrow\left(\theta_{1}, \theta_{2}\right) \rightarrow\left(\theta_{1}, \theta_{2}^{*}\right)$. If, at this time, the input in $B_{00}$ is switched to $u_{\min }$, then the trajectory could return to the starting point. Thus, we will create a periodic orbit that cycles between neighborhoods of the points $\left(x_{1 \varepsilon}, \phi\left(x_{1 \varepsilon}\right)\right)$ and $\left(\theta_{1}, \theta_{2}^{*}\right)$. A suitable qualitative control will be time-varying and use the time constants $T_{\varepsilon}, T_{\alpha}$, and $T_{P 3}$ established, respectively, in Lemmas 5, 3, and 2. Let $\Delta T=T_{\varepsilon}+T_{\alpha}+T_{P 3}$ and define the time-varying control law, with $j \in \mathbb{N}_{0}$ :

$$
\begin{aligned}
& u(t, X(x))= \\
& \begin{cases}1, & \forall t, x \in B_{11} \\
u_{\text {min }}, & \forall t, x \in B_{10} \cup B_{01} \\
u_{\min }, & \forall t \in\left(j \Delta T, j \Delta T+T_{\varepsilon}\right), x \in B_{00} \\
u_{\max }, & \forall t \in\left(j \Delta T+T_{\varepsilon},(j+1) \Delta T\right), x \in B_{00} .\end{cases}
\end{aligned}
$$

Theorem 4 Assume that H1 and H2 hold. The system (4) with control function (13) has a periodic solution passing through $\left(\theta_{1}, \theta_{2}\right)$.

Proof. Since $u=u_{\min }$ in $B_{10} \cup B_{01}$, it is easy to see that any trajectory will eventually reach the (closed) region $\bar{B}_{00}$. Observe that the control (13) in $B_{00}$ consists of applying $u=u_{\text {min }}$ during a time interval of length $T_{\varepsilon}$, and then applying $u=u_{\max }$ during a time interval of length $T_{\alpha}+T_{P 3}$. By Lemma 5 , at the end of time $T_{\varepsilon}$, any trajectory with initial condition in $\bar{B}_{00}$ and $u=u_{\text {min }}$ has reached an $\varepsilon$-neighborhood, $B_{\varepsilon}$, of $\phi_{00, \text { min }}$, and satisfies $x_{1} \leq x_{1 \varepsilon}$. After a time-interval of duration $T_{\varepsilon}$ has elapsed, the control is switched to $u_{\max }$. Since $B_{\varepsilon}$ is above the separatrix $\alpha$, it follows from the application of Lemma 1 and then Lemma 2 that trajectories leaving from $B_{\varepsilon}$ will pass through point P3 after some finite time. An estimate of this time is $T_{\alpha}+T_{P 3}$, as given by Lemmas 3 and 2 . Therefore, after a time $\Delta T=T_{\varepsilon}+T_{\alpha}+T_{P 3}$, all trajectories with an initial condition in $B_{00}$ have passed through P3 and, according to Lemma 4, they are following a sliding mode solution along the segment $x_{1}=\theta_{1}$ towards the point $\left(\theta_{1}, \theta_{2}^{*}\right)$. After an interval $\Delta T$, the control is switched back to $u_{\text {min }}$, and the cycle repeated. Switching the control between $u_{\min }$ and $u_{\max }$ at these regular intervals, allows any trajectory to eventually enter an oscillatory solution that cycles between $B_{\varepsilon}$ and the segment joining $\left(\theta_{1}, \theta_{2}\right)$ and $\left(\theta_{1}, \theta_{2}^{*}\right)$, while passing exactly through P3.

\section{Applicability and extensions}

The control problems studied in the previous sections are, in fact, not trivial problems, even though they are applied to a fairly simple two-dimensional bistable system. This is in part because both 


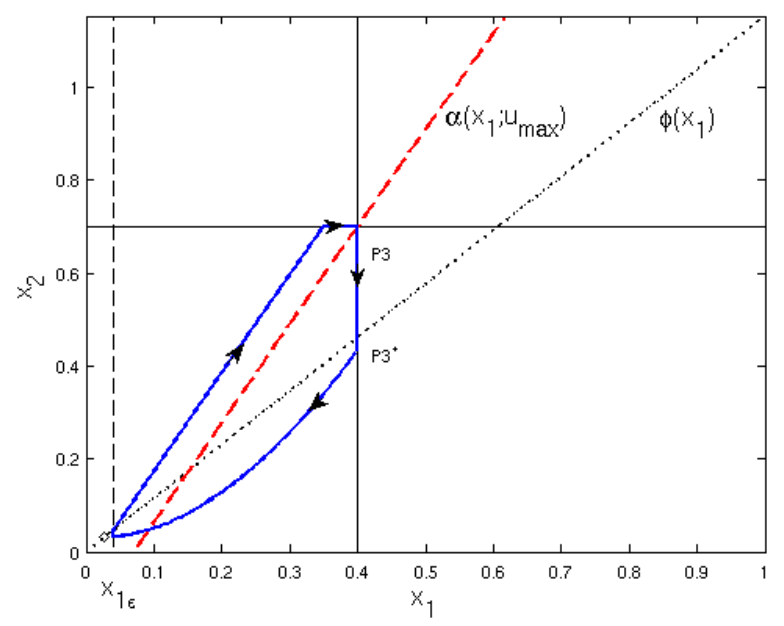

Figure 3: A periodic solution passing through P3, as in Theorem 4. The system can be also controlled to $3^{*}$ (and hence to a neighbourhood of P3), according to Theorem 3.

observations and available inputs are quite restricted and qualitative, which makes classical control techniques impossible to apply, but new biologically-inspired designs are still possible. Indeed, the synthetic circuit designed by Gardner et al. [10], responds to inputs which are very close to our laws. A comparison between our results and the experiments described in Figure 4(a) in [10] is as follows:

1. Suppose GFP fluorescence measures variable $x_{2}$, so that "high state" corresponds to P2: $X_{1}=0$, $X_{2}=1$.

2. It is reasonable to assume that, as the experiment starts, the state is $X_{1}=X_{2}=0$ (genes not expressed).

3. Applying IPTG to the system leads to $X_{2}=1$ : this corresponds to setting $u=u_{\max }$ in $B_{00}, B_{01}$, or equivalently applying Theorem 2 for $t>T_{1}$, since starting orthant is $B_{00}$. Note that $u\left(B_{01}\right)=u_{\max }$ does not qualitatively change the convergence to $\mathrm{P} 2$.

4. Applying next a thermal pulse leads to $X_{2}=0$ : this corresponds to starting from P2 (or $B_{01}$ ) and applying $u=u_{\min }$, or equivalently applying Theorem 1 ; one indeed observes that $x_{2}$ decreases towards 0.

To further emphasize the utility of this study, note that system (3) is one of the motifs that form the basis of regulatory biological/genetic circuits; a double negative feedback loop frequently appears as one of the driving mechanisms in a larger system (the lactose operon [17], the phage $\lambda$ [18], etc.). Some recent analysis techniques can uncover reduced systems which form the core of the original system, under given experimental conditions; moreover, these reduced systems fully represent the asymptotic dynamics of the original system. An example is given in [22], which identifies reduced systems of two or three dimensions representing a set of "active" or operational interactions, under different stimuli. In this context, our results may become extremely useful: suppose system (3) is a reduced model that faithfully reproduces the asymptotic dynamical behaviour of the original larger network, under some specific conditions. It is then reasonable to suppose that the same control laws that drive (3) to a desired state will also drive the full network to a dynamically equivalent state. To illustrate this idea, observe that the results in the previous sections can be used to control the $n$-dimensional positive feedback loop $\left(\dot{x}_{i}=\kappa_{i} s^{+}\left(x_{i-1}, \theta_{i-1}\right)-\gamma_{i} x_{i}\right)$ under the assumption that $n-2$ of the steps have a much "faster" dynamics and that the control is applied on the slower variables : in this case the system reaches a quasi-steady state, where the full system simplifies to the dynamics of the two slower variables.

\section{Conclusion}

This paper addressed the problem of controlling a 2-dimensional PWA system using only qualitative state measurements and qualitative input values, inspired by the biological control of genetic networks. 
Analysis of a single-input bistable system shows that this qualitative control framework can be a powerful tool. In spite of stiff constraints on the inputs, we were able to design control laws that exactly solve the problem of globally asymptotically controlling the system to either of its stable steady states. Control laws were also designed to approximately solve the problem of controlling the system to the unstable steady state, either by stabilizing to a (small) neighborhood of the point or generating a periodic solution passing through the point.

Related problems to be explored in future work include the question of robustness of the control laws. In practice, the switch between two regions of the state space is not sharp and may not be immediately detected in the laboratory. In this case, a description allowing some safety margin in the definition of the inhibition functions may be desirable (as in [4]), by inserting small intermediate regions where the input may take either value.

These controllability problems are of interest for systems which are often subject to data constraints, and where implementation of feedback control laws is a crucial issue. Piecewise constant control laws based on three qualitative values are much easier to implement than a control law which makes use of (often unavailable) detailed quantitative state measurements. Indeed, some of our results are comparable to strategies already used in the laboratory [10], and suggest further possibilities. Notably, control design may be first studied for a reduced system, valid under some specific conditions, and then implemented in the larger original system. The general framework developed in [9] can be used as a starting point to address fine-tuned control problems for higher dimension systems.

\section{References}

[1] S. Azuma, E. Yanagisawa, and J. Imura. Controllability analysis of biosystems based on piecewise affine systems approach. IEEE Trans. Automat. Control, 53:139-152, 2008.

[2] C. Belta, L.C.G.J.M. Habets, and V. Kumar. Control of multi-affine systems on rectangles with applications to hybrid biomolecular networks. In Proc. 41st IEEE Conf. Decision and Control (CDC02), volume 1, pages 534-539, 2002.

[3] R. Casey, H. de Jong, and J.L. Gouzé. Piecewise-linear models of genetic regulatory networks: equilibria and their stability. J. Math. Biol., 52:27-56, 2006.

[4] M. Chaves, T. Eissing, and F. Allgöwer. Bistable biological systems: a characterization through local compact input-to-state stability. IEEE Trans. Automat. Control, 53:87-100, 2008.

[5] M. Chaves, E.D. Sontag, and R. Albert. Methods of robustness analysis for boolean models of gene control networks. IEE Proc. Syst. Biol., 153:154-167, 2006.

[6] H. de Jong, J.L. Gouzé, C. Hernandez, M. Page, T. Sari, and J. Geiselmann. Qualitative simulation of genetic regulatory networks using piecewise linear models. Bull. Math. Biol., 66:301-340, 2004.

[7] R. Edwards, S. Kim, and P. van den Driessche. Control design for sustained oscillation in a two-gene regulatory network. J. Math. Biol., 2010. DOI: 10.1007/s00285-010-0343-y.

[8] M.B. Elowitz and S. Leibler. A synthetic oscillatory network of transcriptional regulators. Nature, 403:335-338, 2000.

[9] E. Farcot and J.L. Gouzé. A mathematical framework for the control of piecewise-affine models of gene networks. Automatica, 44(9):2326-2332, 2008.

[10] T.S. Gardner, C.R. Cantor, and J.J. Collins. Construction of a genetic toggle switch in Escherichia coli. Nature, 403:339-342, 2000.

[11] E.P. Gianchandani, J.A. Papin, N.D. Price, A.R. Joyce, and B.O. Palsson. Matrix formalism to describe functional states of transcriptional regulatory systems. PLoS Comput Biol, 2(8):e101, 2006. 
[12] L. Glass and S.A. Kauffman. The logical analysis of continuous, nonlinear biochemical control networks. J. Theor. Biol., 39:103-129, 1973.

[13] J.L. Gouzé and T. Sari. A class of piecewise linear differential equations arising in biological models. Dyn. Syst., 17(4):299-316, 2002.

[14] L.C.G.J.M. Habets and J. van Schuppen. A control problem for affine dynamical systems on a full-dimensional polytope. Automatica, 40:21-35, 2004.

[15] F. Jacob and J. Monod. Genetic regulatory mechanisms in the synthesis of proteins. J. Mol. Biol., 3:318-356, 1961.

[16] E. Klipp, R. Herwig, A. Howald, C. Wierling, and H. Lehrach. Systems Biology in Practice. Wiley-VCH, Weinheim, 2005.

[17] E.M. Ozbudak, M. Thattai, H.N. Lim, B.L. Shraiman, and A. van Oudenaarden. Multistability in the lactose utilization network of Escherichia coli. Nature, 427:737-740, 2004.

[18] M. Ptashne. A genetic switch: phage $\lambda$ and higher organisms. Cell Press \& Blackwell scientific publications, 1992.

[19] E.D. Sontag. Mathematical Control Theory (2nd ed.). Springer-Verlag, New York, 1998.

[20] E.D. Sontag. Some new directions in control theory inspired by systems biology. Syst. Bio., $1(1): 1-18,2004$.

[21] M. Tigges, T.T. Marquez-Lago, J. Stelling, and M. Fussenegger. A tunable synthetic mammalian oscillator. Nature, 457:309-312, 2009.

[22] L. Tournier and M. Chaves. Uncovering operational interactions in genetic networks using asynchronous boolean dynamics. J. Theor. Biol., 260(2):196-209, 2009.

\section{A Sliding mode solutions}

For self-containment of the paper, here we recall and summarize some facts related to PWA systems, which are given with more detail in [13] and [3]. Consider a 2-dimensional system with variables $(x, y)$, $\dot{x}=f(x, y), \dot{y}=g(x, y)$, with $f, g$ PWA functions. In each regular domain $D$ let $f^{D}$ and $g^{D}$ represent the corresponding vector fields. Although step functions are not defined at threshold points, solutions of the system can still be defined in the sense of Filippov [3], as the solutions of the differential inclusion:

$$
\left(\begin{array}{c}
\dot{x} \\
\dot{y}
\end{array}\right) \in H(x, y)
$$

where $H(x, y)$ is defined on regular domains $D$ as the usual function $H(x, y)=\left(f^{D}(x, y), g^{D}(x, y)\right)^{\prime}$, and on switching domains $D^{s}$ as the convex hull of the vector fields in the neighbouring regular domains

$$
H(x, y)=\overline{\operatorname{co}}\left\{\left(\begin{array}{l}
f^{D}(x, y) \\
g^{D}(x, y)
\end{array}\right): D^{s} \subset \partial D\right\} .
$$

Adopting Definition 4 in [3], a solution of (14) on $[0, T]$ in the sense of Filippov is an absolutely continuous (w.r.t. $t$ ) function $\xi\left(t ; x_{0}\right)$ such that $\xi\left(0 ; x_{0}\right)=x_{0}$ and $d \xi / d t \in H(\xi)$ for almost all $t \in[0, T]$.

Suppose that along one of the thresholds $x=\theta$ (and $\theta_{y}^{a}<y<\theta_{y}^{b}$ always), the vector fields are given as follows:

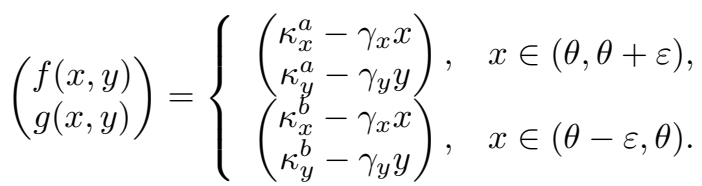


Then, depending on the relative directions, there may be different solutions. The next Lemma states that in some cases the solution can be "normally" continued across a switching domain. The proof follows by showing that, to satisfy the differential inclusion on the contiguous domains, the solutions before and after the $x$ threshold can simply be concatenated.

Lemma 6 Suppose that the vector fields in (15) satisfy: $\left(\kappa_{x}^{a}-\gamma_{x} x\right)\left(\kappa_{x}^{b}-\gamma_{x} x\right)>0$, for all $x \in(\theta-\varepsilon, \theta+$ $\varepsilon)$. Without loss of generality assume that $\kappa_{x}^{a}-\gamma_{x} x>0$. For initial conditions of the form $\left(\theta, y_{0}\right)$ there is a solution in the sense of Filippov, satisfying: $x(t)=\frac{\kappa_{x}^{a}}{\gamma_{x}}+\left(\theta-\frac{\kappa_{x}^{a}}{\gamma_{x}}\right) e^{-\gamma_{x} t}, y(t)=\frac{\kappa_{y}^{a}}{\gamma_{y}}+\left(y_{0}-\frac{\kappa_{y}^{a}}{\gamma_{y}}\right) e^{-\gamma_{y} t}$.

The existence of a solution along a switching domain is next summarized, a particular case which is also known as a sliding mode, where one of the variables remains constant while the other evolves towards a given point determined by the signs of the vector field. This situation appears frequently in the control of the bistable switch.

Lemma 7 Suppose that the vector fields in (15) satisfy $\kappa_{x}^{a}-\gamma_{x} x<0<\kappa_{x}^{b}-\gamma_{x} x$, for all $x \in(\theta-\varepsilon, \theta+\varepsilon)$. For initial conditions of the form $\left(\theta, y_{0}\right)$ there is a solution in the sense of Filippov, satisfying:

$$
x(t) \equiv \theta, \quad y(t)=\frac{\tilde{\kappa}}{\gamma_{y}}+\left(y(0)-\frac{\tilde{\kappa}}{\gamma_{y}}\right) e^{-\gamma_{y} t},
$$

with $\tilde{\kappa}=(1-\beta) \kappa_{y}^{b}+\beta \kappa_{y}^{a}$ and $\beta=\left(\kappa_{x}^{b}-\gamma_{x} \theta\right) /\left(\kappa_{x}^{b}-\kappa_{x}^{a}\right)$, defined on an interval $[0, T)$, where $0<T \leq \infty$. The trajectory evolves towards a point: $(\bar{x}, \bar{y})=\left(\theta, \tilde{\kappa} / \gamma_{y}\right)$. If $\bar{y} \in\left(\theta_{y}^{a}, \theta_{y}^{b}\right)$, then $T=\infty$. If $\bar{y} \notin\left(\theta_{y}^{a}, \theta_{y}^{b}\right)$, then $T<\infty$. In particular,

$$
y(T)= \begin{cases}\theta_{y}^{a}, & \operatorname{sign}\left(\kappa_{y}^{a}-\gamma_{y} y\right)=\operatorname{sign}\left(\kappa_{y}^{b}-\gamma_{y} y\right)<0 \\ \theta_{y}^{b}, & \operatorname{sign}\left(\kappa_{y}^{a}-\gamma_{y} y\right)=\operatorname{sign}\left(\kappa_{y}^{b}-\gamma_{y} y\right)>0,\end{cases}
$$

where the inequalities should hold for all $y \in\left(\theta_{y}^{a}, \theta_{y}^{b}\right)$.

Proof. On the line $x=\theta$ any solution can be written as a convex combination of the vector fields, $0<\beta<1$ :

$$
\begin{aligned}
& \dot{x}=\beta\left(\kappa_{x}^{a}-\gamma_{x} x\right)+(1-\beta)\left(\kappa_{x}^{b}-\gamma_{x} x\right) \\
& \dot{y}=\beta\left(\kappa_{y}^{a}-\gamma_{y} y\right)+(1-\beta)\left(\kappa_{y}^{b}-\gamma_{y} y\right)
\end{aligned}
$$

Given the orientation of the vector fields on $x$, solutions may reach the line $x=\theta$, coming from either side, but they cannot leave it as long as $y \in\left(\theta_{y}^{a}, \theta_{y}^{b}\right)$. Any solution must satisfy $x(t)=\theta$ for $t$ in some time interval. In this case, it makes sense to choose $\dot{x}=0$ when $x=\theta$ (see also [13]). This can be used to solve for the parameter $\beta$ : $\beta=\left(\kappa_{x}^{b}-\gamma_{x} \theta\right) /\left(\kappa_{x}^{b}-\kappa_{x}^{a}\right)$ and obtain a unique solution for $y$, of the form (16), with $\tilde{\kappa}=(1-\beta) \kappa_{y}^{b}+\beta \kappa_{y}^{a}$. As $t \rightarrow \infty$, the $y$ solution tends to $\bar{y}=\tilde{\kappa} / \gamma_{y}$. If $\bar{y} \in\left(\theta_{y}^{a}, \theta_{y}^{b}\right)$, then the trajectory will converge towards $(\bar{x}, \bar{y})$, a fixed point in the sense of Filippov. If $\bar{y} \notin\left(\theta_{y}^{a}, \theta_{y}^{b}\right)$, then the trajectory stops once it reaches one of the endpoints of the interval. If $\operatorname{sign}\left(\kappa_{y}^{a}-\gamma_{y} y\right)=\operatorname{sign}\left(\kappa_{y}^{b}-\gamma_{y} y\right)<0$ (resp. $>0$ ), then all the vectors in the cone imply that $y$ is decreasing (resp., increasing), so that the trajectory reaches one of the endpoints of the $y$ interval in finite time. In particular, note that one has $0>\kappa_{y}^{a}-\gamma_{y} \theta_{y}^{a}>\kappa_{y}^{a}-\gamma_{y} \theta_{y}^{b}$ and $0>\kappa_{y}^{b}-\gamma_{y} \theta_{y}^{a}>\kappa_{y}^{b}-\gamma_{y} \theta_{y}^{b}$, which implies $\bar{y} \leq(1-\beta) \theta_{y}^{a}+\beta \theta_{y}^{a}=\theta_{y}^{a}$. That is, the solution tends to a point below $\theta_{y}^{a}$, so it will stop here. A similar reasoning holds for case (b). 